\title{
Clinical characteristics and survival of colorectal cancer patients in Korea stratified by age
}

\author{
Sun Kyung Baek ${ }^{1}$, Ji Sung Lee ${ }^{2}$, In Gyu Hwang³ , Jong Gwang Kim4, Tae Won Kim5 , Seung Kook Sohn ${ }^{6}$, \\ Mi Yeon Kang7, and Sang-Cheol Lee ${ }^{8}$
}

\begin{abstract}
${ }^{1}$ Department of Internal Medicine, Kyung Hee University Hospital, Seoul; ${ }^{2}$ Clinical Research Center, Asan Medical Center, Seoul; ${ }^{3}$ Department of Internal Medicine, Chung-Ang University Hospital, Seoul; ${ }^{4}$ Department of Oncology/ Hematology, Kyungpook National University Medical Center, Daegu; ${ }^{5}$ Department of Oncology, Asan Medical Center, University of Ulsan College of Medicine, Seoul; ${ }^{6}$ Health Insurance Review and Assessment Service, Wonju; ${ }^{7}$ Quality Assessment Management Division, Health Insurance Review and Assessment Service, Wonju; ${ }^{8}$ Department of Internal Medicine, Soonchunhyang University Cheonan Hospital, Cheonan, Korea
\end{abstract}

Received: February 19, 2019

Revised : October 11, 2019

Accepted: May 20, 2020

\section{Correspondence to}

Sang-Cheol Lee, M.D.

Division of Hematology and

Oncology, Department of Internal Medicine, Soonchunhyang

University Cheonan Hospital, 31

Suncheonhyang 6-gil, Dongnam-

gu, Cheonan 31151, Korea

Tel: +82-41-570-3803

Fax: +82-41-574-5762

E-mail: leptin72@gmail.com

https://orcid.org/0000-0003-

0139-9909
Background/Aims: This nationwide study was undertaken to determine differences in clinicopathologic characteristics and survival of patients with colorectal cancer (CRC) according to age using big data from the Korean National Health Insurance Service (NHIS).

Methods: The NHIS data including quality assessment of CRC by the Health Insurance Review \& Assessment Service in Korea between 2011 and 2014 were analyzed. Based on age, patients were divided into three groups: not-old patients (< 65 ), young-old patients ( 65 to 74 years old) and old-old patients ( $\geq 75$ years old).

Results: We included 71,513 CRC patients. The median follow-up duration was 3.2 years (range, 0.003 to 5.5 ). Male patients constituted $60 \%$. The median age of patients was 65 years (range, 18 to 102). Colon was the cancer site in $59.8 \%$ of not-old patients, $62.9 \%$ of young-old patients, and $66.1 \%$ of old-old patients. Compared to not-old patients, young-old and old-old patients were more likely to be diagnosed with colon adenocarcinoma and well/moderate differentiation or adequate differentiation (all $p<0.001)$. Old patients underwent more emergency operation $(p<$ $0.001)$ and received less adjuvant therapy in stage I-III $(p<0.001)$. The probability of 3-year survival of young-old or old-old patients was worse than that for not-old patients (hazard ratio [HR], 1.55; 95\% confidence interval [CI], 1.46 to 1.64)(HR, 3.19; $95 \%$ CI, 3.03 to 3.37 ).

Conclusions: Old patients with CRC show different histology from younger patients. They are more frequently to have colon as primary lesion. They undergo less adjuvant therapy. Further studies and evidence-based guidelines for older patients with CRC are warranted to improve their outcome.

Keywords: Colorectal cancer; Elderly; Histology; Survival

\section{INTRODUCTION}

The incidence rate for colorectal cancer (CRC) is increasing continuously in Korea. Annual percentage change in age-standardized incidence rates was $5.7 \%$ in men and 4.3\% in women between 1999 and 2012 using the world standard population as a standard [1]. Such increases in CRC incidence in the Korean population might be ex- 
plained by western diet, physical inactivity, high alcohol consumption, and high body mass index and early detection by the National Cancer Screening Program for Medical Aid recipients.

CRC is known as a disease of the elderly. Age is a known risk factor for cancer due to duration of carcinogenesis, vulnerability of aging tissues to environmental carcinogens, and other bodily changes that favor the development and growth of cancer [2]. Age may also influence cancer biology. In Korea, several reports have suggested differences in patterns of CRC incidence according to age, sex, and anatomical locations [3,4] based on histology and recurrence patterns after curative resection for CRC [5]. However, the clinicopathologic patterns and survivals of CRC in the Korean population remain to be investigated.

Advances in treatment options including medical and surgical innovations have resulted in substantial improvement in survival mainly in patients included in clinical trials. Age alone is not a contraindication to cancer treatment. As older and frail patients are under-represented in clinical trials [6], most evidence is derived from "not-old" patients. Most evidence related to treatment of older CRC patients has been pooled from analyses of older patients included in large prospective clinical trials and community-based studies $[7,8]$.

The aim of the current study was to demonstrate clinical characteristics and survival in CRC according to age and cancer stage based on the Korean National Health Insurance (KNHI) service big data analysis.

\section{METHODS}

The Health Insurance Review \& Assessment Service of Korea has been conducting quality assessment of hospitals for major cancers including breast, gastric, liver and CRC to improve the quality of management and treatment for cancer patients since 2012 (data of patients diagnosed at 2011 year). We analyzed the KNHI database of patients admitted to nearly 230 hospitals. These patients underwent quality assessment of CRC between 2012 and 2015. Anatomical subsites were defined based on the International Statistical Classification of Diseases and Related Health Problems, 1oth revision (ICD-10). Data were collected using CRC code (C18, C19, C2O)
The KNHI database contains information regarding age, gender, stage, date of diagnosis, primary site (by surgery code), histology, differentiation (well/moderate/ poor differentiation from 2011 to 2012, differentiation/ no differentiation from 2013 to 2014), emergency operation, number of lymph node dissections, adjuvant radiation, and chemotherapy were collected. Patients were divided into two groups: not-old (<65-year-old) and old ( $\geq 65$-year-old) patients. Old patients were further divided into young-old (65 to 74-year-old) and old-old ( $\geq 75$ -year-old) patients.

Survival was indirectly assessed based on patients' death date recorded in the KNHI service. Outcome variable was overall survival (OS) time starting from the date of diagnosis until either the date of death or the end of the study (until 30 June 2016). This study was approved by the Institutional Review Board of Soonchunhyang University Hospital Cheonan (IRB No. SCHCA-2016-07-008). Informed consent by the patients was waived due to a retrospective nature of our study.

\section{Statistical analysis}

As mentioned above, clinical characteristics and survival of the three age groups were analyzed. Descriptive statistics were computed for all variables as frequencies and percentages for categorical variables using a chi-square test. OS probability according to age or stage was calculated using Kaplan-Meier product limit method. Hazard ratios (HR) and 95\% confidence interval (CI) values of OS were analyzed using the Cox proportional hazards model for age and stage. All statistical analyses were performed using SAS software version 9.4 (SAS Institute, Cary, NC, USA). A two sided $p$ value of less than 0.05 was considered statistically significant.

\section{RESULTS}

\section{Baseline characteristics according to age}

A total of 71,513 patients were enrolled from 2011 to 2014. Median follow-up duration was 3.2 years (range, 0.003 to 5.5). Male patients constituted 60.1\%. Median age of all patients was 65 years (range, 18 to 102). Patients with stage I, II, III, and IV accounted for $22.0 \%$, 29.0\%, 35.6\%, and $12.9 \%$, respectively. According to surgical code, $59.8 \%$ and $37.1 \%$ of patients were diagnosed with colon 
Table 1. Baseline characteristics

\begin{tabular}{|c|c|c|c|c|c|}
\hline Characteristic & $\begin{array}{c}\text { Total } \\
(\mathrm{n}=71,513)\end{array}$ & $\begin{array}{c}18-64 \mathrm{yr} \\
(\mathrm{n}=34,615)\end{array}$ & $\begin{array}{c}65-74 \mathrm{yr} \\
(\mathrm{n}=22,259)\end{array}$ & $\begin{array}{c}\geq 75 \text { yr } \\
(\mathrm{n}=14,639)\end{array}$ & $p$ value \\
\hline \multicolumn{6}{|l|}{ Sex } \\
\hline Male & $42,944(60.1)$ & $21,519(62.2)$ & $13,891(62.4)$ & $7,534(51 \cdot 5)$ & $<0.0001$ \\
\hline Female & $28,569(39 \cdot 9)$ & $13,096(37.8)$ & $8,368(37 \cdot 6)$ & $7,105(48.5)$ & 0.5652 \\
\hline Stage & & & & & $<0.0001$ \\
\hline Stage I & $15,748(22.0)$ & $8,186(23 \cdot 7)$ & $5,235(23.6)$ & $2,327(16.0)$ & \\
\hline Stage II & $20,772(29.0)$ & $8,953(26.0)$ & $6,539(29 \cdot 5)$ & $5,280(36.2)$ & \\
\hline Stage III & $25,456(35 \cdot 6)$ & $12,421(36.0)$ & $7,795(35 \cdot 1)$ & $5,240(36.0)$ & \\
\hline Stage IV & $9,249(12.9)$ & $4,911(14.2)$ & $2,617(11.8)$ & $1,721(11.8)$ & \\
\hline Primary site (by surgery code) & & & & & $<0.0001$ \\
\hline Colon & $42,736(59.8)$ & $19,980(59.1)$ & $13,602(62.9)$ & $9,154(66.1)$ & \\
\hline Rectum & $26,526(37 \cdot 1)$ & $13,804(40.9)$ & $8,031(37.1)$ & $4,691(33 \cdot 9)$ & \\
\hline Histology $(n=30,925)$ & & & & & $<0.0001$ \\
\hline Adenocarcinoma & $29,840(95 \cdot 5)$ & $12,970(96.1)$ & $9,497(97.1)$ & $7,373(96.4)$ & \\
\hline Mucinous adenocarcinoma & $990(3.2)$ & $470(3.5)$ & $267(2.7)$ & $253(3 \cdot 3)$ & \\
\hline Others, signet-ring cell carcinoma, unknown & $95(0.3)$ & $54(0.4)$ & $17(0.2)$ & $24(0.3)$ & \\
\hline Differentiation $(\mathrm{n}=14,047,2011$ to 2012$)$ & & & & & $<0.0001$ \\
\hline Well/moderate differentiation & $13,311(94.8)$ & $5,982(95 \cdot 1)$ & $4,331(95 \cdot 4)$ & $2,998(93.2)$ & \\
\hline Poor differentiation & $579(4.1)$ & $236(3.8)$ & $155(3.4)$ & $188(5.8)$ & \\
\hline Others, unknown & $157(1.1)$ & $72(1.1)$ & $55(1.2)$ & $30(0.9)$ & \\
\hline Emergency operation & & & & & $<0.0001$ \\
\hline Yes & $3,498(4 \cdot 9)$ & $1,220(3.5)$ & $929(4.2)$ & $1,349(9.2)$ & \\
\hline No & $68,015(95.1)$ & $33,395(96.5)$ & $21,330(95.8)$ & $13,290(90.8)$ & \\
\hline Treatment (stage I-III) & & & & & $<0.0001$ \\
\hline Surgery only & $35,989(58.1)$ & $14,406(48.7)$ & $11,334(57 \cdot 9)$ & $10,249(79.8)$ & \\
\hline Surgery + adjuvant Tx (CTx or RT or both) & $25,987(41.9)$ & $15,154(51.3)$ & $8,235(42.1)$ & $2,598(20.2)$ & \\
\hline
\end{tabular}

Values are presented as number (\%).

Tx, treatment; CTx, chemotherapy; RT, radiotherapy.

cancer and rectal cancer, respectively. Based on histology, $95.5 \%, 3.2 \%$, and $0.3 \%$ of patients had adenocarcinoma, mucinous adenocarcinoma, and other type or signet-ring cell type, respectively. Among the total, $4.9 \%$ patients underwent emergency operation and $58.1 \%$ patients with stage I-III received adjuvant therapy (radiation, chemotherapy, or both) after surgery (Table 1 ).

Of a total of 71.513 patients, 34,615 (48.4\%), 22,259 (31.5\%), and 14,639 (20.5\%) were not-old, young-old, and old-old patients, respectively. Females accounted for 37.8\%, $37.6 \%$, and $48.5 \%$ of not-old, young-old, and old-old patients, respectively. Stage IV patients constituted 14.2\%, $11.8 \%$, and $11.8 \%$ of not-old, young-old and old-old pa- tients. Colon was the cancer site in $59.8 \%$ of not-old patients, $62.9 \%$ of young-old patients, and $66.1 \%$ of old-old patients. Emergency operation was conducted in 3.5\% of not-old patients, $4.2 \%$ of young-old patients, and $9.2 \%$ of old-old patients. In patients with stage I-III cancer, $51.3 \%$ of not-old patients, $42.1 \%$ of young-old patients, and $20.2 \%$ of old-old patients received adjuvant therapy including radiation or chemotherapy or both (Table 1).

\section{Survival according to age and stage}

Three-year survival probabilities of not-old, young-old, and, old-old patients were $87.8 \%, 82.5 \%$, and $67.9 \%$, respectively (Table 2 and Supplementary Fig. 1). Young-old 
Table 2. Survival rate according to age

\begin{tabular}{lccccccc}
\hline Age, yr & $\begin{array}{c}\text { Total no. of } \\
\text { patients }\end{array}$ & $\begin{array}{c}\text { Total no. } \\
\text { of events }\end{array}$ & $\begin{array}{c}\text { Survival } \\
\text { probability at } \\
\text {-year, \% (95\% CI) }\end{array}$ & $\begin{array}{c}\text { Survival } \\
\text { probability at } \\
\text { 3-year, \% (95\% CI) }\end{array}$ & $\begin{array}{c}\text { Survival } \\
\text { probability at } \\
\text { 5-year, \% (95\% CI) }\end{array}$ & HR(95\% CI) & p value \\
\hline $18-64$ & 34,615 & 4,731 & $96.4(96.2,-96.6)$ & $87.8(87.4-88.1)$ & $82.3(81.8-82.9)$ & Reference & \\
$65-74$ & 22,259 & 4,310 & $93.5(93.2-93.8)$ & $82.5(81.9-83.0)$ & $75.1(74.3-75.8)$ & $1.48(1.44-1.53)$ & $<0.0001$ \\
$\geq 75$ & 14,639 & 5,034 & $85.6(85.1-86.2)$ & $67.9(67.1-68.7)$ & $56.2(55.1-57.3)$ & $3.03(2.95-3.12)$ & $<0.0001$ \\
\hline
\end{tabular}

CI, confidence interval; HR, hazard ratio.

Table 3. Survival rate according to stage

\begin{tabular}{lccccccc}
\hline Stage & $\begin{array}{c}\text { Total no. of } \\
\text { patients }\end{array}$ & $\begin{array}{c}\text { Total no. } \\
\text { of events }\end{array}$ & $\begin{array}{c}\text { Survival } \\
\text { probability at } \\
\text { 1-year, \% (95\% CI) }\end{array}$ & $\begin{array}{c}\text { Survival } \\
\text { probability at } \\
\text { 3-year, \% (95\% CI) }\end{array}$ & $\begin{array}{c}\text { Survival } \\
\text { probability at } \\
\text { 5-year, \% (95\% CI) }\end{array}$ & HR(95\% CI) & $p$ value \\
\hline I & 15,748 & 878 & $98.2(98.0-98.4)$ & $95.4(95.1-95.7)$ & $91.9(91.3-92.6)$ & Reference & \\
II & 20,772 & 2,614 & $95.9(95.6-96.2)$ & $89.2(88.7-89.6)$ & $82.8(82.1-83.5)$ & $2.37(2.20-2.56)$ & $<0.0001$ \\
III & 25,456 & 5,074 & $94.4(94.1-94.7)$ & $82.3(81.8-82.8)$ & $73.8(73.1-74.6)$ & $3.90(3.63-4.19)$ & $<0.0001$ \\
IV & 9,249 & 5,406 & $76.1(75.2-77.0)$ & $42.9(41.8-44.0)$ & $30.4(29.1-31.7)$ & $16.58(15.43-17.81)$ & $<0.0001$ \\
\hline
\end{tabular}

CI, confidence interval; HR, hazard ratio.

and old-old patients had worse 3-year survival probabilities than not-old patients ([HR, 1.48; 95\% CI, 1.44 to 1.53], [HR, 3.03; 95\% CI 2.95 to 3.12], respectively).

Three-year survival probability of patients with stage I, II, III, and IV cancer were 95.4\%, 89.2\%, 82.3\%, 42.9\%, respectively (Table 3 and Supplementary Fig. 2). For notold patients, 5 -year survival probabilities of those with stage II, stage III, and stage IV cancer were worse than those with stage I caner ([HR, 2.44; 95\% CI, 2.07 to 2.88], [HR, 5.09; 95\% CI, 4.39 to 5.90], [HR, 32.21; 95\% CI, 27.88 to 37.21], respectively) (Table 4). For young-old patients, 5 -year survival probabilities of those with stage II, stage III, and stage IV cancer were worse than those with stage I cancer ([HR, 2.08; 95\% CI, 1.82 to 2.37], [HR, 3.88; 95\% CI, 3.43 to 4.38 ], [HR, 17.36; $95 \%$ CI, 15.35 to 19.63], respectively) (Table 4). For old-old patients, 5-year survival probabilities of those with stage I, stage II, stage III, and stage IV cancers were also worse than those with stage I cancer ([HR, 1.67; 95\% CI, 1.49 to 1.87], [HR, 2.78; 95\% CI, 2.49 to 3.10], [HR, 8.51; 95\% CI, 7.58 to 9.56]) (Table 4).

\section{DISCUSSION}

CRC is the third most common cancer and the fourth most common cause of cancer death in Korea. The incidence rate of CRC with thyroid cancer continues to increase in both men and women [1]. Although the incidence of CRC in men was higher than that in women in this study, the proportion of females in the old-old group was higher than in the not-old or the young-old age group. Similar results have been reported from Korea and America, showing stationary male-to-female incidence rate ratios after 70 s with decreases after $80 \mathrm{os}[4,9]$. Although reasons for higher incidence in men are not completely understood yet, etiological factors related to complex interactions between sex hormones and risk factor exposure and differences in screening behavior between gender might play a role [10].

Aging is associated with a progressive depletion of functional reserve of multiple organs and systems, increased prevalence of comorbid conditions, and waning social and economic resources at a time when they are most needed. A final definition of "old" is still unavailable. According to World Health Organization, most countries have accepted a chronological age of 65 years as a definition of older age [11]. Considering that the prevalence of comorbidity and functional dependence increase progressively with age, older patients can be classified into three categories: (1) "young old" patients 
Table 4. Survival rate according to age and stage

\begin{tabular}{|c|c|c|c|c|c|c|c|}
\hline Stage & $\begin{array}{l}\text { Total no. } \\
\text { of patients }\end{array}$ & $\begin{array}{l}\text { Total no. } \\
\text { of events }\end{array}$ & $\begin{array}{c}\text { Survival } \\
\text { probability at } \\
\text { 1-year, \% }(95 \% \mathrm{CI})\end{array}$ & $\begin{array}{c}\text { Survival } \\
\text { probability at } \\
\text { 3-year, \% (95\% CI) }\end{array}$ & $\begin{array}{c}\text { Survival } \\
\text { probability at } \\
\text { 5-year, \% (95\% CI) }\end{array}$ & $\mathrm{HR}(95 \% \mathrm{CI})$ & $p$ value \\
\hline \multicolumn{8}{|c|}{ Not-old patients $(\mathrm{n}=34,471)$} \\
\hline Total & 34,471 & 4,700 & $96.4(96.2-96.6)$ & $87.8(87.4-88.2)$ & $82.4(81.8-82.9)$ & & \\
\hline Stage I & 8,186 & 200 & $99 \cdot 3(99.1-99.5)$ & $98.1(97.8-98.4)$ & $96.3(95.7-96.9)$ & Reference & \\
\hline Stage II & 8,953 & 520 & $98.7(98.4-98.9)$ & $95 \cdot 2(94.7-95 \cdot 7)$ & $92.0(91.2-92.7)$ & $2.44(2.07-2.88)$ & $<0.0001$ \\
\hline Stage III & 12,421 & 1,465 & $98.1(97.8-98.3)$ & $90.1(89.5-90.6)$ & $83.6(82.7-84.5)$ & $5.09(4.39-5.90)$ & $<0.0001$ \\
\hline \multicolumn{8}{|c|}{ Young-old patients $(\mathrm{n}=\mathbf{2 2 , 1 8 6 )}$} \\
\hline Total & 22,186 & 4,282 & $93.5(93.2-93.9)$ & $82.5(82.0-83.0)$ & $75.1(74.4-75 \cdot 9)$ & & \\
\hline Stage I & 5,235 & 305 & $98.1(97.7-98.5)$ & $95.0(94.4-95.7)$ & $91.5(90.4-92.6)$ & Reference & \\
\hline Stage II & 6,539 & 761 & $96.5(96.1-97.0)$ & $90.1(89.3-90.9)$ & $83.7(82.4-85.0)$ & $2.08(1.82-2.37)$ & $<0.0001$ \\
\hline Stage III & 7,795 & 1,612 & $94.5(94.0-95.1)$ & $81.9(81.0-82.8)$ & $72.4(71.0-73.7)$ & $3.88(3.43-4.38)$ & $<0.0001$ \\
\hline Stage IV & 2,617 & 1,604 & $74.0(72.3-75.7)$ & $39.7(37.7-41.7)$ & $28.4(26.0-30.8)$ & $17.36(15 \cdot 35-19.63)$ & $<0.0001$ \\
\hline \multicolumn{8}{|c|}{ Old-old patients $(\mathrm{n}=14,568)$} \\
\hline Total & 14,568 & 4,990 & $85.7(85.1-86.3)$ & $68.0(67.2-68.8)$ & $56.4(55.2-57.5)$ & & \\
\hline Stage I & 2,327 & 373 & $94.8(93.9-95.7)$ & $86.5(85.0-88.0)$ & $76.3(73.7-79.0)$ & Reference & \\
\hline Stage II & 5,280 & 1,333 & $90.4(89.6-91.2)$ & $77.6(76.4-78.8)$ & $65.3(63.4-67.2)$ & $1.67(1.49-1.87)$ & $<0.0001$ \\
\hline Stage III & 5,240 & 1,997 & $85.6(84.7-86.6)$ & $64.1(62.8-65.5)$ & $51.7(49.8-53.7)$ & $2.78(2.49-3.10)$ & $<0.0001$ \\
\hline Stage IV & 1,721 & 1,287 & $59.4(57.1-61.7)$ & $25.6(23.3-27.8)$ & $15.5(12.7-18.3)$ & $8.51(7.58-9.56)$ & $<0.0001$ \\
\hline
\end{tabular}

CI, confidence interval; HR, hazard ratio.

aged between 65 and 75 years; (2) old-old patients in the 76 to 85 years of age; and (3) "oldest old" patients older than 85 years of age $[2,12]$. The purpose of this study was to demonstrate differences in the characteristics and survival of patients with CRC according to age including "not-old" patients. In this study, old patients aged more than 75 years were not as high as $20.5 \%$. Therefore, we divided old age into only two categories: (1) "young-old" patients aged between 65 and 74 years; and (2) "old-old" patients aged more than 75 years. Increased age probably introduces population variations in life expectancy related to non-cancer mortality rather than CRC risk. In this study, less difference in survival probability was observed in old patients than in not-old patients.

As for primary site, the proportion of rectal cancer based on surgical code decreased from young to older age groups. A recent population-based analysis in the US has demonstrated a steady decline in rectal cancer in patients aged 50 years and older, with the most notable decline found in patients older than 75 years [13], consistent with our results. Although the present study did not compare the ratio of left to right colon, many studies have reported that right tumor location increases while rectal cancer decreases with age [13-15]. Unlike primary site which showed consistent trend with age, the proportion of mucinous adenocarcinoma and poor differentiated cancer is not observed by age. Histologically, the proportion of poorly differentiated adenocarcinoma in patients older than 75 years was about 6\%. It was higher than that in young-old group.

Young-old and old-old patients increasingly underwent emergency operation and received fewer adjuvant treatments such as chemotherapy or radiation or both. Elderly patients had more right side cancer with obstruction or advanced stage. Patients who presented as an emergency and required immediate operative intervention are known to have higher morbidity and overall mortality $[16,17]$. These patients also show a relatively poor survival due to less successful resection and more advanced disease at presentation [18]. In this study, patients undergoing emergency operation constituted 4.9\% and $9.2 \%$ in young-old and old-old group, respectively. These percentages were much lower than those (22\% to $25 \%$ ) in previous reports from Sweden and UK 
$[17,19]$. In Korea, all adults aged 50 years or older were invited for CRC screening as a national cancer screening program starting in 2004. Colonoscopy is inexpensive and readily accessible. It is widely conducted in outpatient clinics and private screening centers equipped with skilled endoscopists. Screening at a young age and easy accessibility of colonoscopy may result in fewer emergency operations. Age could affect the decision of adjuvant treatment in practice. Actually, several adjuvant trials such as X-ACT and the Multicenter International Study of Oxaliplatin/5-Fluorouracil/Leucovorin in the Adjuvant Treatment of Colon Cancer (MOSAIC) trial included patients up to 75 years $[20,21]$. However some studies have suggested that administration of adjuvant treatment could induced moderate increment of benefit for patients $\geq 70$ or 75 years of age $[12,22]$. Clear treatment guidelines for older patients are lacking. However, proper selection of elderly patients is the key to the benefit of adjuvant chemotherapy through appropriate geriatric assessment. Comprehensive geriatric assessment (CGA) is a multidisciplinary, in-depth evaluation to assess the risk of morbidity, mortality, and life expectancy of older persons. It also helps clinicians develop coordinated plans and optimal treatment for the elderly with cancer. Cancer-specific geriatric assessment as a brief type of CGA for older cancer patients has been developed. It has shown its usefulness for predicting chemotherapy-related toxicity in older patients with cancer $[23,24]$.

This study has some limitations. First, this study performed retrospective big data analysis based on the existing NHIS database. Therefore, it has limitation in that comorbidities, performance status, and geriatric evaluation of patients could not be performed. In addition, the cause of cancer death was unavailable due to indirect data based on patient's death date reported in KNHI service. Natural mortality rate could influence survival of patients in older age groups. Analysis of relative survival would require access to the country's life table. Our analysis was limited by inadequate data availability. Additionally, missing data related to histology and differentiation prevented a comparative analysis. Finally, comparison between right and left colon was not included in this analysis. Nevertheless, this study was meaningful in that it compared clinical characteristics and survival rates of Korean CRC patients according to clinical age and cancer stage based on big data including over 70,000 patients.

In conclusion, more than half of CRC in Korea is diagnosed in individuals aged 65 years or older. The proportion of rectal cancer decreased from young to older age groups. Compared to not-old patients, old patients showed different histology and survival. Elderly patients increasingly underwent emergency operation and received fewer adjuvant treatments. Prospective studies and evidence-based guidelines are needed for older patients with CRC to improve their outcomes.

\section{KEY MESSAGE}

1. More than half of patients with colorectal cancer (CRC) in Korea occurs at age 65 or older.

2. The different histology and poor survival are shown in old patients.

3. This is useful data of clinical practice and helpful to understand the epidemiology of Korean patients with CRC by big data analysis.

\section{Conflict of interest}

No potential conflict of interest relevant to this article was reported.

\section{Acknowledgments}

This study was supported by the grant from the Soonchunhyang University research and by the Korean Cancer Study Group (KCSG). This study was also supported by a grant from the National R\&D Program for Cancer Control, Ministry of Health and Welfare, Republic of Korea (1720150).

\section{REFERENCES}

1. Jung KW, Won YJ, Kong HJ, et al. Cancer statistics in Korea: incidence, mortality, survival, and prevalence in 2012. Cancer Res Treat 2015;47:127-141.

2. Balducci L. Management of cancer in the elderly. Oncology (Williston Park) 2006;20:135-143.

3. Jung KW, Park S, Kong HJ, et al. Cancer statistics in Korea: incidence, mortality, survival, and prevalence in 2009. Cancer Res Treat 2012;44:11-24.

4. Shin A, Kim KZ, Jung KW, et al. Increasing trend of col- 
orectal cancer incidence in Korea, 1999-2009. Cancer Res Treat 2012;44:219-226.

5. Seo SI, Lim SB, Yoon YS, et al. Comparison of recurrence patterns between $\leq 5$ years and $>5$ years after curative operations in colorectal cancer patients. J Surg Oncol 2013;108:9-13.

6. Talarico L, Chen G, Pazdur R. Enrollment of elderly patients in clinical trials for cancer drug registration: a 7-year experience by the US Food and Drug Administration. J Clin Oncol 2004;22:4626-4631.

7. Folprecht G, Seymour MT, Saltz L, et al. Irinotecan/fluorouracil combination in first-line therapy of older and younger patients with metastatic colorectal cancer: combined analysis of 2,691 patients in randomized controlled trials. J Clin Oncol 2008;26:1443-1451.

8. Goldberg RM, Tabah-Fisch I, Bleiberg H, et al. Pooled analysis of safety and efficacy of oxaliplatin plus fluorouracil/leucovorin administered bimonthly in elderly patients with colorectal cancer. J Clin Oncol 2006;24:40854091.

9. Siegel R, Desantis C, Jemal A. Colorectal cancer statistics, 2014. CA Cancer J Clin 2014;64:104-117.

10. Murphy G, Devesa SS, Cross AJ, Inskip PD, McGlynn KA, Cook MB. Sex disparities in colorectal cancer incidence by anatomic subsite, race and age. Int J Cancer 2011;128:1668-1675.

11. Kowal P, Dowd JE.Definition of an older person. Proposed working definition of an older person in Africa for the MDS Project [Internet]. Geneva $(\mathrm{CH})$ : World Health Organization, 2001 [cited 2020 Nov 30]. Available from: https://www.academia.edu/19488959/Definition_of_an_ older_person_Proposed_working_definition_of_an_older_person_in_Africa_for_the_MDS_Project.

12. Berretta M, Di Benedetto F, Di Francia R, et al. Colorectal cancer in elderly patients: from best supportive care to cure. Anticancer Agents Med Chem 2013;13:1332-1343.

13. Bailey CE, Hu CY, You YN, et al. Increasing disparities in the age-related incidences of colon and rectal cancers in the United States, 1975-2010. JAMA Surg 2015;150:17-22.

14. Itatani Y, Kawada K, Sakai Y. Treatment of elderly patients with colorectal cancer. Biomed Res Int 2018;2018:2176056.

15. Siegel RL, Miller KD, Fedewa SA, et al. Colorectal cancer statistics, 2017. CA Cancer J Clin 2017;67:177-193.

16. Anderson JH, Hole D, McArdle CS. Elective versus emergency surgery for patients with colorectal cancer. $\mathrm{Br} \mathrm{J}$ Surg 1992;79:706-709.

17. Sjo OH, Larsen S, Lunde OC, Nesbakken A. Short term outcome after emergency and elective surgery for colon cancer. Colorectal Dis 2009;11:733-739.

18. Runkel NS, Schlag P, Schwarz V, Herfarth C. Outcome after emergency surgery for cancer of the large intestine. Br J Surg 1991;78:183-188.

19. Kodeda K, Nathanaelsson L, Jung B, et al. Population-based data from the Swedish Colon Cancer Registry. Br J Surg 2013;100:1100-1107.

20. Andre T, Boni C, Navarro M, et al. Improved overall survival with oxaliplatin, fluorouracil, and leucovorin as adjuvant treatment in stage II or III colon cancer in the MOSAIC trial. J Clin Oncol 2009;27:3109-3116.

21. Scheithauer W, McKendrick J, Begbie S, et al. Oral capecitabine as an alternative to i.v. 5-fluorouracil-based adjuvant therapy for colon cancer: safety results of a randomized, phase III trial. Ann Oncol 2003;14:1735-1743.

22. Sanoff HK, Carpenter WR, Sturmer T, et al. Effect of adjuvant chemotherapy on survival of patients with stage III colon cancer diagnosed after age 75 years. J Clin Oncol 2012;30:2624-2634.

23. Hurria A, Gupta S, Zauderer M, et al. Developing a cancer-specific geriatric assessment: a feasibility study. Cancer 2005;104:1998-2005.

24. Hurria A, Togawa K, Mohile SG, et al. Predicting chemotherapy toxicity in older adults with cancer: a prospective multicenter study. J Clin Oncol 2011;29:3457-3465. 


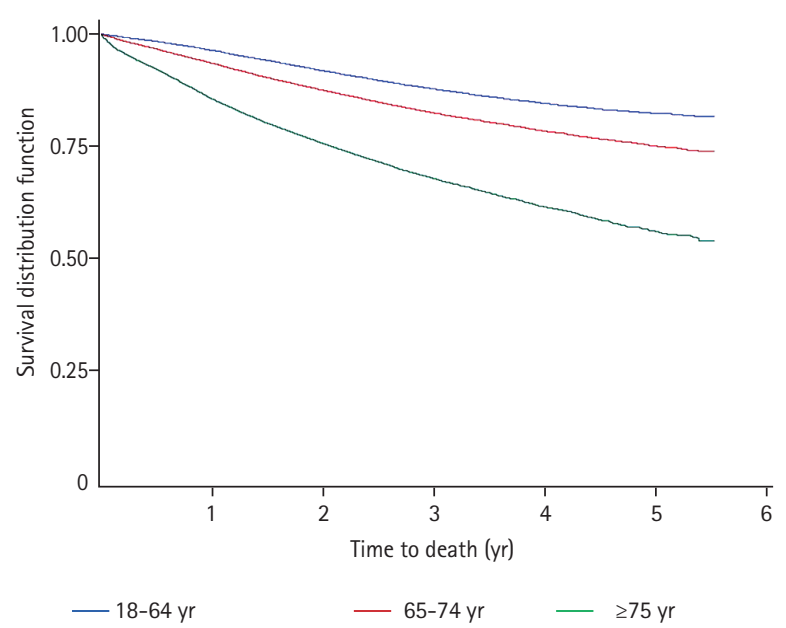

Supplementary Figure 1. Survival rate according to age groups. 


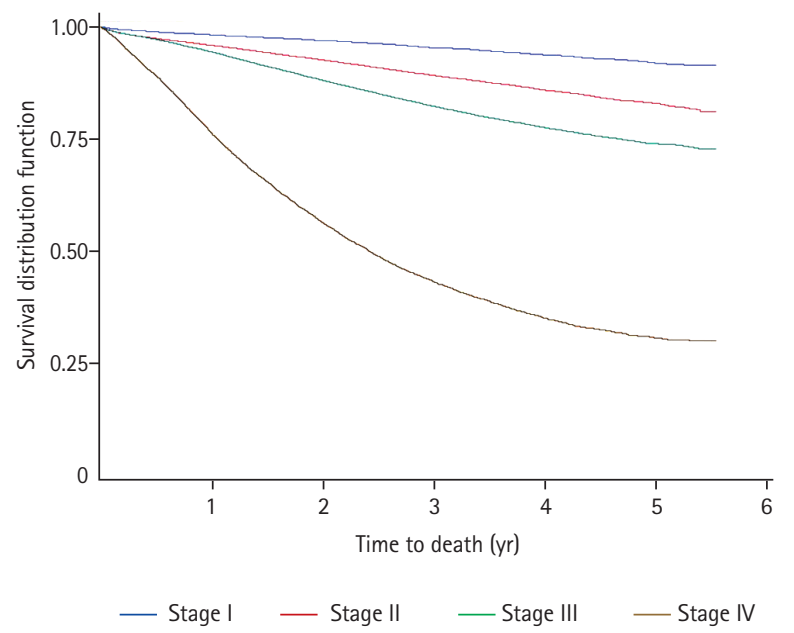

Supplementary Figure 2. Survival rate according to cancer stage. 